\title{
Development and Evaluation of a Web-Based System to Learn Skills for Removing Personal Protective Equipment for Highly Infectious Diseases
}

\author{
Shota SATO ${ }^{\mathrm{a}, 1}$, Sachiko IIJIMA ${ }^{\mathrm{a}}$ and Yoko MURANAKA ${ }^{\mathrm{b}}$ \\ ${ }^{a}$ Graduate School of Health Care and Nursing, Juntendo University, Japan \\ ${ }^{\mathrm{b}}$ Faculty of Nursing, Shumei University, Japan
}

\begin{abstract}
We developed a web-based learning system for healthcare workers to learn the skills for removing personal protective equipment for highly infectious diseases. The system was implemented on a trial basis and healthcare workers evaluated its usability and their motivation to learn. Usability was similar to that of typical systems. Motivation scores were high, indicating confidence and satisfaction with the system.
\end{abstract}

Keywords. Web-based learning system, removing, personal protective equipment, highly infectious disease, motivation, usability

\section{Introduction}

To prevent secondary infection with highly infectious diseases such as Ebola virus disease, healthcare workers (HCWs) are trained to use full-body personal protective equipment (PPE) and to remove it safely. However, no studies have examined the strategies used for retaining these skills in the long term [1]. The objective of this study was to develop a web-based learning system for HCWs to learn the skills for removing PPE and to evaluate the usability of the system and their motivation to learn it.

\section{Methods}

We developed the learning system based on the ADDIE model-analysis, design, development, implementation, and evaluation. First, we analyzed actions that could cause contamination and identified precautions taken when putting on and removing PPE based on literature. Second, we designed the system with the goal of teaching and reinforcing skills for removing PPE properly. Third, we developed the learning system using Moodle 3.6.3 [2]. Data were entered in HTML, and MySQL was accessed from PHP on the server side. The data were mainly images, videos, and PDF files. Fourth, we implemented the system on a trial basis. Fifth, we evaluated its usability and participants'

\footnotetext{
${ }^{1}$ Corresponding Author, Shota Sato, Doctoral student, Graduate School of Health Care and Nursing, Juntendo University, 2-1-1 Takasu, Urayasu, Chiba 279-0023, Japan; E-mail: m3116013@juntendo.ac.jp.
} 
motivation to learn. We recruited participants through network sampling. The participants participated voluntarily and were asked to use the system for one month. Usability was evaluated using the System Usability Scale (SUS), which converted the obtained data to a scale of $0-100$ points [3], to calculate the participants' average SUS scores. Motivation was evaluated using an original 20 -item questionnaire based on the five categories of the ARCS-V model-attention, relevance, confidence, satisfaction, and volition [4]. Answers to each question were rated on a 5-point Likert scale.

\section{Results}

Seven individuals who had experience of putting on and removing PPE for highly infectious diseases used the system. Five of them answered the questionnaire. The average SUS score was 69.0 points. The means (standard deviations) for the ARCS-V model were 3.6 (0.69), 3.7 (1.22), 4.2 (0.89), 4.1 (0.76), and 3.4 (1.27) for attention, relevance, confidence, satisfaction, and volition, respectively.

\section{Conclusions}

The learning system developed in this study had the same mean usability score as those reported previously (68 points) [5], suggesting that its usability was similar to that of typical systems. Overall, the system was deemed helpful in motivating learning. Improvements to the volition element should help to sustain and reinforce learning.

\section{Acknowledgements}

The authors thank all the participants in this study.

\section{References}

[1] Verbeek JH. Personal protective equipment for preventing highly infectious diseases due to exposure to contaminated body fluids in healthcare staff. Cochrane Database of Systematic Reviews. 2019;7:CD011621.

[2] Moodle.org. [Internet]. 2020 [cited 2020 Mar 25]. Available from: https://moodle.org

[3] Brooke J. SUS: A “quick and dirty” usability scale. Usability Evaluation in Industry. 1996;189-194.

[4] Keller JM. Challenging in learner motivation: a holistic, integrative model for research and design on learner motivation. The 11th International Conference on Education Research. 2010;1-18.

[5] Sauro J, Lewis JR. Quantifying the user experience: Practical statistics for user research, 2nd, editors. Cambridge: Morgan Kaufmann; 2016. p. 204-205. 